\title{
Proportional Retarded Controller to Stabilize Underactuated Systems with Measurement Delays: Furuta Pendulum Case Study
}

\author{
T. Ortega-Montiel, ${ }^{1}$ R. Villafuerte-Segura, ${ }^{1}$ C. Vázquez-Aguilera, ${ }^{2}$ and L. Freidovich ${ }^{3}$ \\ ${ }^{1}$ Research Center on Information Technology and Systems, Hidalgo State University, Pachuca, HGO, Mexico \\ ${ }^{2}$ Control System Team, Ålö AB, Umeå, Sweden \\ ${ }^{3}$ Department of Applied Physics and Electronics, Umeå University, Umeå, Sweden
}

Correspondence should be addressed to R. Villafuerte-Segura; villafuerte@uaeh.edu.mx

Received 15 June 2017; Accepted 16 August 2017; Published 10 December 2017

Academic Editor: Libor Pekař

Copyright ( 2017 T. Ortega-Montiel et al. This is an open access article distributed under the Creative Commons Attribution License, which permits unrestricted use, distribution, and reproduction in any medium, provided the original work is properly cited.

\begin{abstract}
The design and tuning of a simple feedback strategy with delay to stabilize a class of underactuated mechanical systems with dead time are presented. A linear time-invariant (LTI) model with time delay of fourth order and a Proportional Retarded (PR) controller are considered. The PR controller is shown as an appealing alternative to the application of observer-based controllers. This paper gives a step forward to obtain a better understanding of the effect of output delays and related phenomena in mechatronic systems, making it possible to design resilient control laws under the presence of uncertain time delays in measurements and obtain an acceptable performance without using a derivative action. The Furuta pendulum is a standard two-degrees-of-freedom benchmark example from the class of underactuated mechanical systems. The configuration under study includes an inherent output delay due to wireless communication used to transmit measurements of the pendulum's angular position. Our approach offers a constructive design and a procedure based on a combination of root loci and Mikhailov methods for the analysis of stability. Experiments over a laboratory platform are reported and a comparison with a standard linear state feedback control law shows the advantages of the proposed scheme.
\end{abstract}

\section{Introduction}

Underactuated mechatronic systems have been a subject of intensive research during the past three decades, where partial feedback linearization, normal forms, and energybased methods have offered successful results as well as breakthrough paradigms [1-9]. Experiments with pendulums having one or more degrees of underactuation play an important role in control theory. One important challenge concerning the construction of such equipment is how to send the measurements from the encoders located in the underactuated links without adding restrictions to the movement; one possible solution is wireless communication [10, 11]. Nowadays, wireless sensor networks are a feasible option when mobility and flexibility are requirements. However, when the sensor signals are transmitted through a wireless network, the time-delay phenomena are unavoidable, representing an important as well as an open challenge [11-13].
Time delays in measurements are often neglected. Moreover, when only positions are instrumented, the corresponding velocities are often required to be reconstructed, either with an observer or with the use of on-line differentiators or filters (see, e.g., $[14,15])$. It is well known that noise, time delays, sampling, and unmodeled dynamics deteriorate the performance of such estimators. Hence, it is of relevance to understand the effect of time delays in measurements as well as the potential of such delays as a second degree of freedom for control design (see $[16,17])$. For this aim, a PR controller design will be presented together with the Mikhailov stability approach. Furthermore, a Furuta pendulum case study, where the underactuated variable is subject to an inherent output delay due to wireless signal transmission, is presented. The analysis of time delays in control systems is not a new topic; particularly, the addition of time delays in the control design has been studied for many years (see, e.g., [18-27]). In particular, the introduction of time delays in the control 
law avoids the use of techniques for estimating the derivative action, which is not an easy task under the presence of noise and delays in measurements and increases the cost and complexity of the overall controller design and tuning of the gains [16]. On the other hand, the implementation of a retarded control law requires only memory registers and is relatively simple to program, since it is based on a sum of a proportional signal and the corresponding delayed one. However, the stability analysis of a closed-loop system with a retarded feedback control law requires dealing with infinitedimensional systems even if the initial plant model is not. This analysis is studied in the framework of two approaches: time domain and frequency domain $[28,29]$. In the first approach, the analysis is based on the well-known Lyapunov's criteria using linear matrix inequalities (LMIs) via convex optimization. However, this approach provides only sufficient conditions of stability or stabilization, which are usually conservative for some systems, while for other systems the LMIs are unfeasible. Besides, in our experience for similar problems, the parameters tuning by a frequency method using LMIs sometimes is not feasible. In the second approach, the analysis is based on the root locus of its corresponding characteristic equation in the complex plane. Unlike the temporal approach, the frequency approach can provide necessary and sufficient conditions that are not conservative. In [30-32], some numerical methods to approximate the roots of these quasi-polynomial or analytic functions are displayed. Furuta pendulum is a well-known academic example that is used as a benchmark for the study of underactuated mechanical systems $[33,34]$. Recently, in [17], the control design of a PR controller for the stabilization of Furuta pendulum was introduced; however, the effect of the inherent delay, present in the unactuated variable, was not studied. In contrast to the previous work [17], this paper presents a more detailed analysis for the tuning of control gains; also new experiments and graphs are presented, as well as experimental tests of robustness under external disturbances. In addition, the organization of the document is better and more natural; also a more comprehensive bibliographic review is performed and the introduction presents a better justification of the topic of study. Besides, to the best of our knowledge, the stability analysis including the inherent time delay as well as the enhancement of the PR control parameters has not been presented for Furuta pendulum. In addition, the Mikhailov procedure [35] is described and formalized for the present case study; concerning application of the method to systems with delay, see, for example, [36, 37]. The proposed control design is composed of two elements: a PD controller for the actuated variable and PR controller for the stabilization of the unactuated link. The main difference with respect to other works is that the stability analysis incudes two delays, one is inherent of the wireless communication and the other is introduced in the control law for stabilization purposes. Moreover, the time derivative of the unactuated variable or its on-line estimation is not required in the proposed control law. Thus, the characteristic equation analyzed is an analytical function or quasi-polynomial with two transcendental terms of the form

$$
\begin{aligned}
Q\left(s, \alpha, \beta, k_{p}, k_{r}, \tau, \tau_{2}\right) \\
=P_{1}\left(s, k_{p}, \alpha, \beta\right)+P_{2}\left(s, k_{p}\right) e^{-\tau s} \\
\quad+P_{3}\left(s, k_{r}\right) e^{-\left(\tau+\tau_{2}\right) s},
\end{aligned}
$$

where $\tau_{2}$ is the inherent delay of the angular position measurements due to the wireless communication, $P_{i}(\cdot), i=$ $1,2,3$, are polynomials with real coefficients, $k_{p}, k_{r}, \tau$ are the parameters of PR controller, and $\alpha, \beta$ are the parameter of PD controller. The first stability analysis is using the Dpartition method [38] and the root continuity property [39, 40]. A growing body of literature has analyzed the stability of quasi polynomials (see [23, 26, 41-44]). Particularly in [26], the delay phenomena have been studied for a fully actuated cart-pendulum system, introducing a full-state feedback controller with feedforward term, assuming that all velocities and positions are measurable.

The present paper focuses on the delay phenomena in an underactuated system called Furuta pendulum. The main result introduces a methodology for the optimal selection of the controller gains and a procedure based on Mikhailov method for the study of complete stability under the presence of measurement delays. Here, it is assumed that only angular positions are available for measurement. For comparison purposes, a design of a state feedback controller, which includes the on-line estimation of velocities, is presented. All the proposed algorithms are verified with simulations and experiments over a laboratory test bench, obtaining the desired performance.

The outline of this paper is distributed as follows. In Section 2, the mathematical model of the Furuta pendulum is introduced. The proposed control design is presented in Section 3. Experimental results are found in Section 4. The conclusions of this study are drawn in Section 5.

\section{Mathematical Model}

The Furuta pendulum test bench consists of a passive pendulum attached to the end of an actuated rotating arm as shown in Figure 1. The coordinates of the centers of mass of both links are given by $\left(x_{1}, y_{1}, z_{1}\right)$ $=\left(l_{1} \cos \phi, l_{1} \sin \phi, 0\right)$ and $\left(x_{2}, y_{2}, z_{2}\right)=\left(L_{1} \cos \phi-\right.$ $\left.l_{2} \sin \phi \sin \theta, L_{1} \sin \phi+l_{2} \cos \phi \sin \theta, l_{2} \cos \theta\right)$, where $\theta$ is the angular position of the pendulum and $\phi$ is the angle of the rotational arm; and both variables represent the generalized coordinates that describe the motion. The following notation is used: $m_{1}$ is the mass of the arm, $m_{2}$ is the pendulum mass, $J_{1}$ and $J_{2}$ are the moments of inertia of the arm and of the pendulum, respectively, $L_{1}$ and $L_{2}$ are the lengths of the arm and the pendulum, respectively, and $g$ is the acceleration due to gravity. Note that with the chosen coordinated system the unstable equilibrium point is located at $(\theta, \dot{\theta}, \phi, \dot{\phi})=(0,0,0,0)$. Note that the equilibrium does not depend on $\phi$ and every real value of $\phi$ corresponds to an unstable equilibrium. A model of the Furuta pendulum can be obtained applying the Euler-Lagrange formalism (see, e.g., [45-47]). The corresponding Lagrangian is given by $\mathscr{L}=T-V$, with $T=(1 / 2) m_{1}\left(\dot{x}^{2}+\dot{y}^{2}\right)+(1 / 2) m_{2}\left(\dot{x}_{2}^{2}+\dot{y}_{2}^{2}+\dot{z}_{2}^{2}\right)$ 

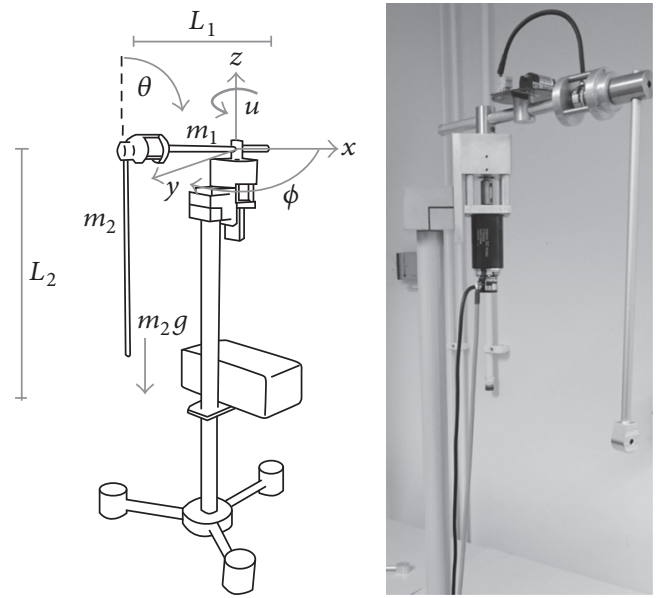

FIGURE 1: Scheme of the Furuta pendulum model by [10].

$+(1 / 2) J_{1} \dot{\phi}^{2}+(1 / 2) J_{2} \dot{\theta}^{2}$ and $V=m_{2} g l_{2} \cos \theta$ leading to the following expression:

$$
M \ddot{q}+(C+D) \dot{q}+h=f,
$$

where the related terms are $q=\left[\begin{array}{l}\theta \\ \phi\end{array}\right], M=\left[\begin{array}{cc}m_{11} & m_{12} \\ m_{12} & m_{22}\end{array}\right], h=$ $\left[\begin{array}{c}0 \\ \kappa u\end{array}\right], C=\left[\begin{array}{cc}0 & c_{12} \\ c_{21} & c_{22}\end{array}\right]$, and $D=\left[\begin{array}{cc}d_{11} & 0 \\ 0 & d_{22}\end{array}\right]$. The elements of the inertia matrix are $m_{11}=J_{2}+m_{2} l_{2}^{2}, m_{12}=m_{2} L_{1} l_{2} \cos \theta$, and $m_{22}=J_{1}+m_{1} l_{1}^{2}+m_{2}\left(L_{1}^{2}+l_{2}^{2} \sin ^{2} \theta\right)$; the Coriolis matrix is formed by $c_{12}=-m_{2} l_{2}^{2} \sin \theta \cos \theta \dot{\phi}, c_{21}=m_{2} l_{2}^{2} \sin \theta \cos \theta \dot{\phi}-$ $m_{2} l_{2} L_{1} \sin \theta \dot{\theta}$, and $c_{22}=m_{2} L_{2}^{2} \sin \theta \cos \theta \dot{\theta}$; the parameters of viscous friction are included in matrix $D$. For the particular laboratory test bench in Figure 1, static friction does not play an important role, because it is small and bounded, and it is neglected in the plant model.

The external torque that allows the control of the arm is produced by a DC motor. The motor induces a torque $M_{a}=$ $c_{a} \kappa_{i} u=\kappa u$, where $u$ is the normalized input voltage of the PWM amplifier, $\kappa_{i}$ is constant, and $\kappa=c_{a} \kappa_{i}$.

There are two control problems commonly associated with the Furuta pendulum: stabilization around one of the two equilibrium points and the swing-up $[48,49]$. In order to study the inherent delay due to the wireless communication together with the design of a retarded control law, here we are going to focus on the problem of stabilization.

In this context, the equation of motion (2) can be linearized around the unstable equilibrium point $(\theta, \dot{\theta}, \phi, \dot{\phi})=$ $(0,0,0,0)$, obtaining the following controllable linear system:

$$
\begin{aligned}
\ddot{\theta}(t) & =a \theta(t)+b \dot{\theta}(t)+c \dot{\phi}(t)+d u(t), \\
\ddot{\phi}(t) & =e \theta(t)+f \dot{\theta}(t)+g \dot{\phi}(t)+h u(t), \\
y\left(t, t-\tau_{2}\right) & =\left[\theta\left(t-\tau_{2}\right), \phi(t)\right]^{T},
\end{aligned}
$$

where $y\left(t, t-\tau_{2}\right)$ is the available output subject to communication delay $\tau_{2}$. The total delay $\tau_{2}$ involved in the sensor communication can be divided into three parts: the communication delay from sensor to controller, the computation
TABLE 1: Physical parameters of Furuta pendulum.

\begin{tabular}{lc}
\hline Mass of the horizontal rod $\left(m_{1}\right)$ & $0.431 \mathrm{~kg}$ \\
Length of the horizontal rod $\left(L_{1}\right)$ & $0.262 \mathrm{~m}$ \\
Distance to center of gravity $\left(l_{1}\right)$ & $0.131 \mathrm{~m}$ \\
Moment of inertia of the rod $\left(J_{1}\right)$ & $0.012 \mathrm{~kg} \mathrm{~m}$ \\
Mass of the pendulum $\left(m_{2}\right)$ & $0.128 \mathrm{~kg}$ \\
Length of the pendulum $\left(L_{2}\right)$ & $0.47 \mathrm{~m}$ \\
Distance to center of gravity $\left(l_{2}\right)$ & $0.185 \mathrm{~m}$ \\
Moment of inertia of pendulum $\left(J_{2}\right)$ & $0.0035 \mathrm{~kg} \mathrm{~m}^{2}$ \\
Friction on $\theta\left(d_{11}\right)$ & $0.0032 \mathrm{~kg} \mathrm{~m}^{2} / \mathrm{s}$ \\
Friction on $\phi\left(d_{22}\right)$ & $0.004 \mathrm{~kg} \mathrm{~m}^{2} / \mathrm{s}$ \\
Motor constant $(\kappa)$ & $1.07 \mathrm{rad} \mathrm{kg} \mathrm{m} / \mathrm{s}^{2}$ \\
\hline
\end{tabular}

delay at the controller, and the communication delay from controller to actuator. Here, any delay from the sensor to the controller is assumed to be small relative to the sampling period, since short delays are typical for optical encoders. Also, the computation delay is relatively small compared with the communication delay that normally is affected by the physical distance between the communication nodes. As a result, $\tau_{2}$ is modeled as a static delay within a bounded set; that is, $\tau_{2} \in\left[0, \tau_{2}^{*}\right]$. The other system parameters are $a=$ $37.377, b=-0.515, c=0.142, d=-35.42, e=-8.228$, $f=0.113, g=-0.173$, and $h=43.28$. Here, the physical parameters of the experimental platform given in Table 1 are employed.

\section{Controller Design and Tuning}

Using classical techniques, stabilization of the Furuta pendulum requires measurements of variables $\theta$ and $\phi$ and the estimation of the corresponding time derivatives $\dot{\theta}$ and $\dot{\phi}$ (see, e.g., $[33,48,49])$. However, when wireless communication is considered, delays in the measurement deteriorate the accuracy of estimation of time derivatives. The system under study includes two encoders for measurements of $\theta$ and $\phi$, where the communication for $\theta$ is via a wireless link. Since the wireless communication introduces a variable time delay in the measurement, the estimation of time derivative for variable $\theta$ is not a trivial task and we aim to avoid this by using a PR control action. The control methodology includes the design of a PR controller to stabilize the passive degree of freedom associated with $\theta$ together with a lowgain PD controller for variable $\phi$. The D-partition method, root locus, and method of Mikhailov are implemented for gain design and stability analysis. Some preliminaries of Mikhailov method and second-order PR controllers are given in Appendices A and B, respectively.

3.1. Control Scheme. Consider the following control law:

$$
u(t)=U\left(t-\tau_{2}\right)+\alpha \phi(t)+\left(\beta-\frac{c}{d}\right) \dot{\phi}(t),
$$

where $U\left(t-\tau_{2}\right)=k_{p} \theta\left(t-\tau_{2}\right)-k_{r} \theta\left(t-\tau-\tau_{2}\right) ; \tau_{2}$ represents the inherent delay of the angular position measurements due to the wireless communication, and $k_{p}, k_{r}$, and $\tau$ are parameters 
of design. For application of this control law, a method for derivative estimation is necessary for variable $\phi$; in this case, either a high-gain observer or a super twisting differentiator can been used in order to obtain $\dot{\phi}$ (see $[15,50]$ ). Thus, the closed-loop system, (3)-(5), is

$$
\begin{aligned}
\ddot{\theta}(t)= & a \theta(t)+b \dot{\theta}(t)+d k_{p} \theta\left(t-\tau_{2}\right) \\
& -d k_{r} \theta\left(t-\tau_{2}-\tau\right)+d \alpha \phi(t)+d \beta \dot{\phi}(t), \\
\ddot{\phi}(t)= & h \alpha \phi(t)+(g+h \beta-(h c) /(d)) \dot{\phi}(t)+e \theta(t) \\
& +f \dot{\theta}(t)+h k_{p} \theta\left(t-\tau_{2}\right) \\
& -h k_{r} \theta\left(t-\tau-\tau_{2}\right),
\end{aligned}
$$

and its representation in state space is given as follows:

$$
\dot{x}(t)=A_{0} x(t)+A_{1} x\left(t-\tau_{2}\right)+A_{2} x\left(t-\tau-\tau_{2}\right),
$$

where

$$
\begin{aligned}
x & =[\theta, \dot{\theta}, \phi, \dot{\phi}], \\
A_{0} & =\left[\begin{array}{cccc}
0 & 1 & 0 & 0 \\
a & b & d \alpha & d \beta \\
0 & 0 & 0 & 1 \\
e & f & h \alpha & g-\frac{h c}{d}+h \beta
\end{array}\right], \\
A_{1} & =\left[\begin{array}{cccc}
0 & 0 & 0 & 0 \\
d k_{p} & 0 & 0 & 0 \\
0 & 0 & 0 & 0 \\
h k_{p} & 0 & 0 & 0
\end{array}\right], \\
A_{2} & =\left[\begin{array}{cccc}
0 & 0 & 0 & 0 \\
-d k_{r} & 0 & 0 & 0 \\
0 & 0 & 0 & 0 \\
-h k_{r} & 0 & 0 & 0
\end{array}\right] .
\end{aligned}
$$

Thus, the characteristic quasi polynomial of system (7) is

$$
\begin{aligned}
& q\left(s, k_{p}, k_{r}, \tau, \tau_{2}, \alpha, \beta\right) \\
& =\operatorname{det}\left\{s I-A_{0}-A_{1} e^{-s \tau_{2}}-A_{2} e^{-s\left(\tau+\tau_{2}\right)}\right\}=s^{4}+\left(\frac{h c}{d}\right. \\
& -h \beta-b-g) s^{3}+\left((b h-f d) \beta-h \alpha+b g-\frac{b h c}{d}\right. \\
& \left.-a-d k_{p} e^{-s \tau_{2}}+d k_{r} e^{-s\left(\tau+\tau_{2}\right)}\right) s^{2}+((a h-d e) \beta \\
& +(b h-f d) \alpha+a g-\frac{a h c}{d}+k_{r}(h c-g d) e^{-s\left(\tau+\tau_{2}\right)} \\
& \left.+k_{p}(g d-h c) e^{-s \tau_{2}}\right) s+(a h-d e) \alpha .
\end{aligned}
$$

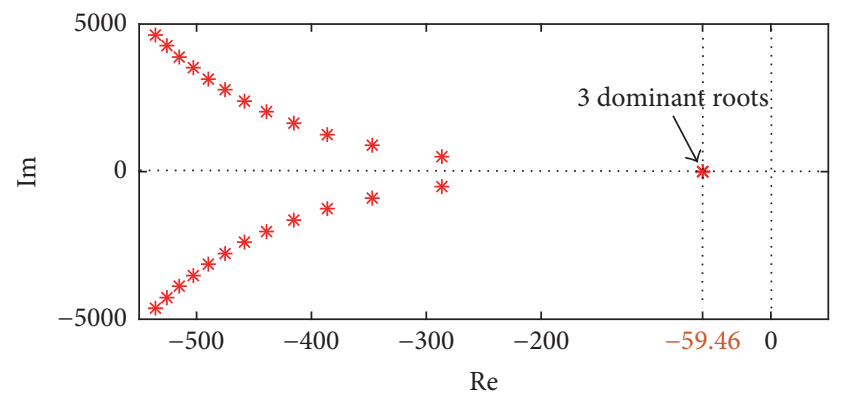

FIGURE 2: Root of quasi polynomial (11).

Note that, for $\alpha=\beta=0$ and $\tau_{2}=0$, it reduces to

$$
\begin{aligned}
q\left(s, k_{p}, k_{r}, \tau, 0,0,0\right)= & \left(s^{2}-b s-a-d k_{p}+d k_{r} e^{-s \tau}\right) \\
& \times\left(s^{2}+\left(\frac{h c}{d}-g\right) s\right) .
\end{aligned}
$$

Clearly, the first factor of the above quasi polynomial is identical to the one for a decoupled one-degree-of-freedom mechanical system under a PR feedback (see (A.3) from Appendix A). Then, as a first step, let us assume small values for $\alpha, \beta$, and $\tau_{2}$, proceeding with the design of $k_{p}, k_{r}$, and $\tau$ with the help of Lemmas A.1 and A.2 given in Appendix B, placing some of the roots into the optimal locations for the reduced system. Lemmas A.1 and A.2 bring $\sigma$-stability to the dynamics of the variable $\theta$. Then, choosing the right values for $\alpha$ and $\beta$, one should not expect $\sigma$-stability for the full fourth-order system but may expect asymptotic stability under sufficiently small and appropriately chosen $\alpha$ and $\beta$, which appears only in the terms associated with $A_{0}$ in (9). This is expected due to continuous dependence of the roots of a quasi polynomial on the coefficients and will be quantified in the next section with the analysis of root locus.

As a second step, the Mikhailov analysis will provide the stability conditions under the presence of an inherent sufficiently small delay $\tau_{2}$.

3.2. Tuning and Stability Analysis. In this section, the tuning of the controller gains proposed in the previous section and an analysis of stability of closed-loop system (7) with $\tau_{2}=0$ are presented.

Consider the second-order quasi polynomial, roots of which cover all the roots of (10) except for $s=0$ and $s=$ $g-h c / d$ :

$$
p\left(s, k_{p}^{*}, k_{r}^{*}, \tau^{*}\right)=s^{2}-b s-a-d k_{p}^{*}+d k_{r}^{*} e^{-s \tau^{*}}
$$

where $k_{p}^{*}, k_{r}^{*}$, and $\tau^{*}$ are tuned for the reduced system (see Lemma A.2). This choice provides three dominant roots in the same point at $-\sigma^{*}$, as can be seen in Figure 2. Suppose that $\alpha$ and $\beta$ are small and $\tau_{2}=0$; then quasi polynomial (9) has five dominant roots, three close to $-\sigma^{*}$ and two close to zero, as can be seen in Figure 3. Therefore, system (7) is close to be marginally stable.

To determine stability of quasi polynomial (9), in Figure 4 , a root locus is presented. Here, $\alpha$ and $\beta$ are varied. 


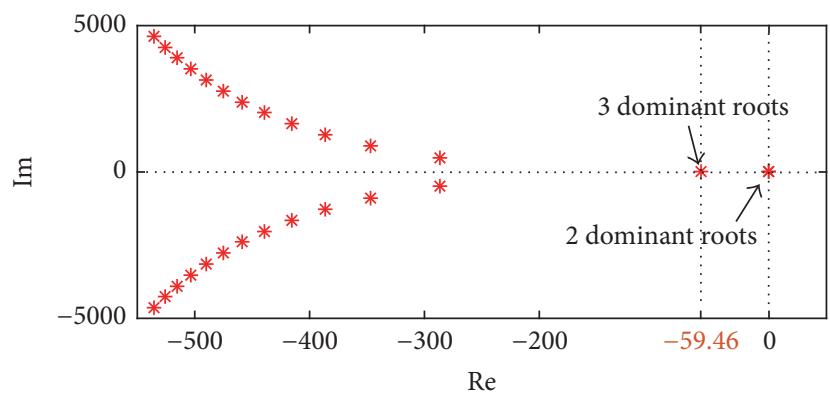

Figure 3: Roots of (9) when $\alpha=\beta=\tau_{2}=0$.

Observe that if $\alpha=0$ and $\beta$ is varied or $\beta=0$ and $\alpha$ is varied, then quasi polynomial (9) remains unstable. The case where $\alpha \neq 0$ and $\beta \neq 0$ can be handled with the same method; however, for some combination of values, it can be exhaustive to find the stability condition or quasi polynomial (9) can turn unstable. Meanwhile, if the value of $\alpha=\beta$ is increased simultaneously, then quasi polynomial (9) can be stabilized relatively easier.

Next, the root locus of quasi polynomial (9) is presented when $\alpha=\beta>0$ are simultaneously varied as can be seen in Figure 5. Here, two of the three dominant roots in $-\sigma^{*}$ are moved to the right and one root is moved to the left, while the two dominant roots at zero and at $g-h c / d \approx 0$ are moved to the left. Clearly, there is a breaking point $\gamma^{*}$ such that quasi polynomial (9) becomes unstable; this occurs when $\gamma^{*} \approx 0.9$. Thus, the stability of system (3) in closed loop with control law (5) is ensured, when $\alpha=\beta \in\left(0, \gamma^{*}\right)$. The above analysis enables a proper selection of the gains $\alpha$ and $\beta$ such that quasi polynomial (9) is stable. Without loss of generality, the gains have been set at $\alpha=\beta=0.5$. Here, the five dominant roots are $s_{1}=-130.3, s_{2,3}=-15.85 \pm 39 i$, and $s_{4,5}=-0.358 \pm 0.78 i$ (see roots marked with blue asterisk in Figure 5). Note that if $\alpha=\beta<0$, then quasi polynomial (9) is unstable.

3.3. Robustness Analysis under the Presence of $\tau_{2}>0$. Now, Mikhailov's criterion [35] to corroborate the stability under the presence of $\tau_{2} \neq 0$ and $\alpha=\beta=0.5$ is implemented (see Appendix B). For this purpose, consider the next closed curve in the complex plane:

$$
C=C_{0}+C_{1}
$$

where $C_{0} \subset \mathbb{C}$ is the interval $[-r, r]$ on the imaginary axis and $C_{1} \subset \mathbb{C}$ is a semicircle of radius $r$ in the right-half complex plane, where $r$ is sufficiently large (we take $r=$ 100). Mikhailov's criterion is used to analyze robustness when $\tau_{2} \in\left[0, \tau_{2}^{*}\right]$. In Figures 6(a) and 6(b), one clockwise turn and one counterclockwise turn around zero are depicted. In Figures 6(c), 6(d), and 6(e), two clockwise turns and two counterclockwise turns around zero are observed. It should be mentioned that such behavior is verified for $\tau_{2}=$ $0.004, \ldots, 0.007$. Nevertheless, the figures have been omitted to save the space. Thus, due to continuous dependence of the roots on the parameter $\tau_{2}$ and due to the above observations, it can be concluded that the change of argument of quasi polynomial (9) is $N=0$ when $\tau_{2} \in[0,0.008]$. However, in
Figure 6(f), two clockwise turns and one counterclockwise turn around zero are observed when $\tau_{2}^{*} \approx 0.009$. Here, $N=1$. Therefore, quasi polynomial (9) remains stable if $\tau_{2} \in[0.008]$.

On the other hand, in Figure 7, the root locus of quasi polynomial (9) when $\tau_{2}=0.008$ and $\alpha=\beta \in[0,0.9]$ is shown. Observe that (9) remains stable if $\tau_{2}=0.008$ and $\alpha=\beta \in[0,0.524]$; and it is unstable if $\tau_{2}=0.008$ and $\alpha=\beta \in[0.524,0.9]$.

Therefore, we have the following result.

Proposition 1. Consider a quasi polynomial of the form (9) and a positive constant $\sigma>-b$. Then, the control law (5) stabilizes a fourth-order system of the form (6) if

$$
\begin{aligned}
& k_{p}^{*}=-\frac{\left(2\left(a+\sigma^{2}+\sigma b\right)+b^{2}\right)}{(2 d)}, \\
& \tau^{*}=\frac{(2 \sigma+b)}{\left(\sigma^{2}+b \sigma-a-d k_{p}^{*}\right)}, \\
& k_{r}^{*}=-\frac{(2 \sigma+b)}{\left(d \tau^{*} e^{\sigma \tau^{*}}\right)} ;
\end{aligned}
$$

and $\alpha, \beta$, and $\tau_{2}$ are such that $\alpha=\beta \in\left(0, \gamma^{*}\right)$ and $\tau_{2} \in\left[0, \tau_{2}^{*}\right)$. Here, $k_{p}^{*}$ follows from (A.5) and $\gamma^{*}$ is given in Section 3.2; $\tau_{2}^{*}=$ 0.008 .

\section{Experiments}

The experimental results were obtained in the Robotics and Control Lab at the Department of Applied Physics and Electronics, Umeå University, Umeå, Sweden. The system used for the experiments is from the PendCon Advanced series [10] with the configuration for rotary pendulum or Furuta pendulum (see Figure 1). In this hardware setup, signals from the encoders with resolution of 4096 pulses per revolution are transmitted wirelessly, and for the actuated link the motor MAXON RE40 $(40 \mathrm{~mm})$ is used, which is a graphite brushless DC motor $(150 \mathrm{~W})$ with nominal voltage of $24 \mathrm{~V}$. The real-time controller is on a dedicated target PC that communicates with the host PC; it has one PCI slot for the encoder card and four interfaces for RS-232 signals; this PC has Intel Core i5 processor with real-time workshop; the host-target environment is a PC with MATLAB real-time workshop compiler installed on Intel Core i5 processor with Window 7. Both communicate with the Conversion Unit via a Matlab-compatible Quatech RS-232 card. The control system is developed in MATLAB R2015a/Simulink and downloaded to the target PC.

The control goal is to stabilize the pendulum at its unstable equilibrium point $\left(\theta^{*}=0\right)$, that is, vertically pointing up. For comparison purposes, a state feedback controller is designed: $u_{\mathrm{sf}}=K x(t)$ with $x(t)=[\theta(t), \dot{\theta}(t), \phi(t), \dot{\phi}(t)]^{T}$. In order to have a reasonable comparison, the gain $K$ is designed such that the poles in closed loop of the linearized system are located at $-15.85 \pm 39 i$ and $-0.3581 \pm 0.78 i$. Note that these values correspond with the dominant complex conjugate poles obtained with the PR controller. In Figure 8, the performance of regulation of the angular positions $\theta(t)$ 

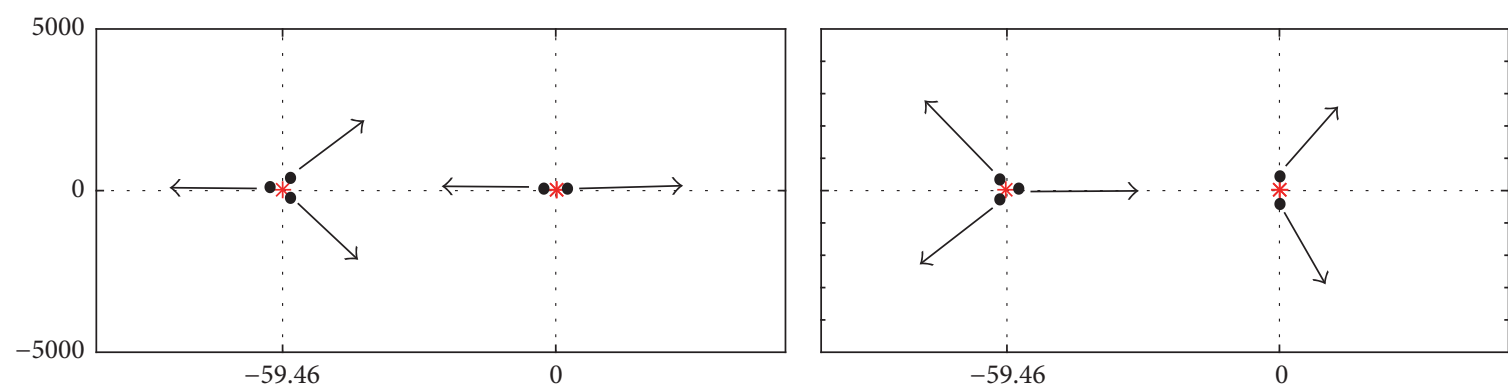

- $\alpha<0, \beta=0$

* $\alpha=\beta=0$

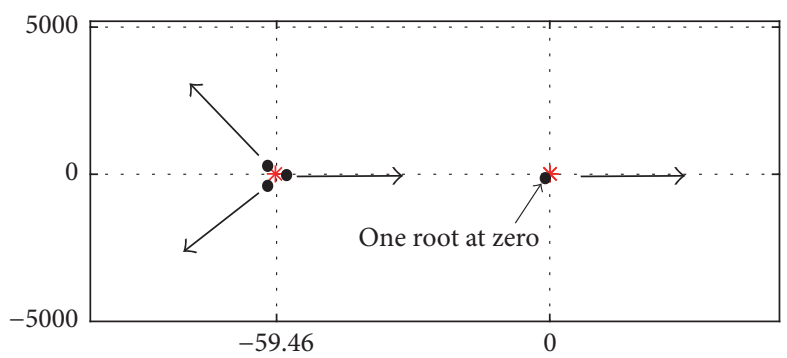

- $\alpha>0, \beta=0$

* $\alpha=\beta=0$

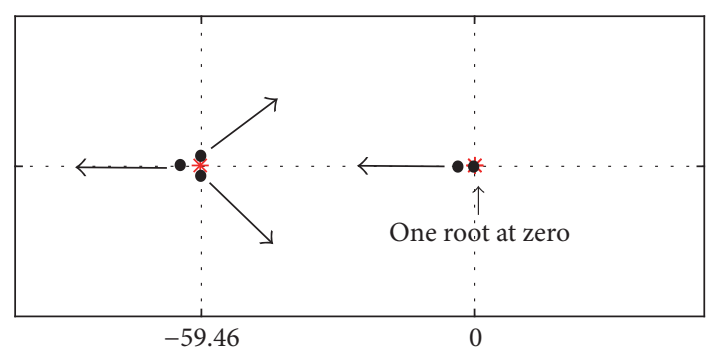

$-\alpha=0, \beta<0$
* $\alpha=\beta=0$

- $\alpha=0, \beta>0$

* $\alpha=\beta=0$

Figure 4: Root locus of (9) when $\tau_{2}=0$ and $\alpha$ and $\beta$ are varied.

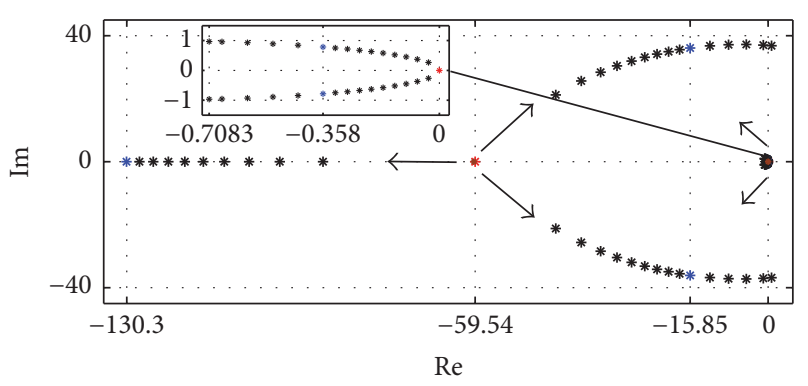

FIgURE 5: Root locus of (9) when $\tau_{2}=0$ and $\alpha=\beta \in[0,0.9]$.

and $\phi(t)$ with the PR and state feedback controller are presented. Here, the initial position is close to the unstable equilibrium point $\theta^{*}$. Figure 9 presents the control signals.

The design and tuning of the controller are done as follows:

(1) Consider a system of the form (3)-(4) in closed loop with a control law of the form (5) and its corresponding quasi polynomial of 4 th order $q\left(s, k_{p}, k_{r}\right.$, $\left.\tau, \tau_{2}, \alpha, \beta\right)$, specified in (9).

(2) Factor the quasi polynomial of 4 th order in one quasi polynomial of 2 nd order and one polynomial of 2 nd order, when $\alpha=\beta=0$ and $\tau_{2}=0$ (see (10)):

$$
q\left(s, k_{p}, k_{r}, \tau, 0,0,0\right)=p\left(s, k_{p}, k_{r}, \tau\right) f(s) .
$$

(3) Use Lemma A.2 to $\sigma$-stabilize the quasi polynomial of 2nd order and obtain the parameters $\left(k_{p}^{*}, k_{r}^{*}, \tau^{*}\right)$ of the PR controller (see (11)):

$$
p\left(s, k_{p}, k_{r}, \tau\right)=p\left(s, k_{p}^{*}, k_{r}^{*}, \tau^{*}\right) .
$$

(4) Obtain $\alpha, \beta$, and $\tau_{2}$ such that quasi polynomial (9) remains stable, as depicted in Figures 6 and 7.

Remark 2. It is important to remark that there is an inherent unknown delay $\tau_{2}>0$, in the measurement of pendulum position; that is, at time $t$, we obtain $\theta\left(t-\tau_{2}\right)$. However, if $\tau_{2}$ remains inside a small range, stability is still preserved as we have shown.

In order to compute the estimation of the first derivative for both position variables, $\theta$ and $\phi$, two methods were explored: high gain and super twisting differentiators (see $[15,50])$.

These methods are designed to be used on-line. For comparison purposes, it is obvious that better velocity estimation can be achieved with an off-line method when both previous and future values of the position are used. Then, we propose to postprocess the measured position to obtain an off-line estimation. For this purpose, the measured signal is fitted with a smoothing spline and next the off-line estimation of velocity is obtained as an analytical differentiation of the spline (see, e.g., [51], page 194). Using this off-line estimation as the true value of the velocity, we compare the performances of high gain (with different gains: HG1 and HG2) and super twisting (ST) differentiators, computing the estimation errors 


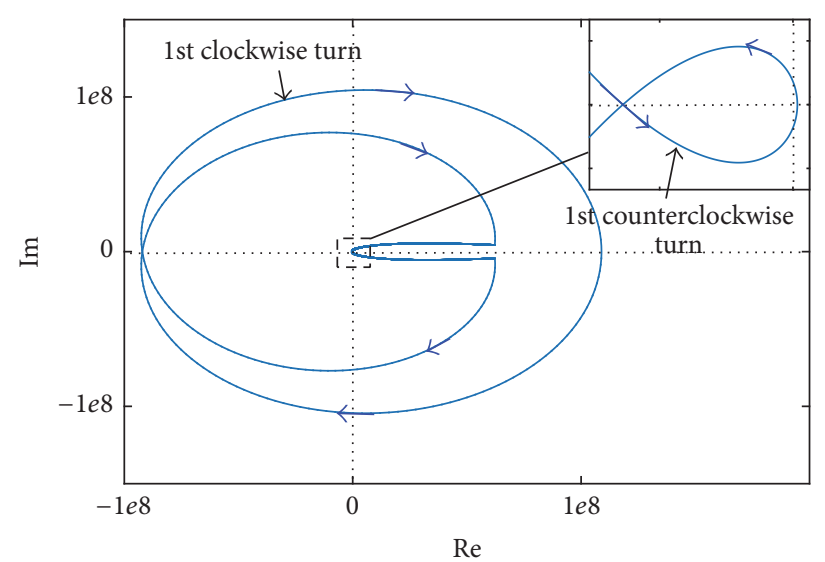

(a) $\tau_{2}=0$

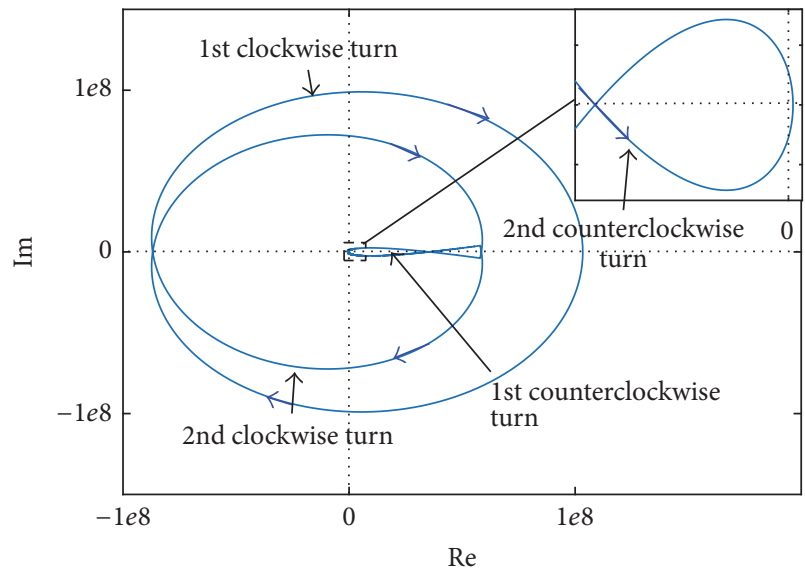

(c) $\tau_{2}=0.002$

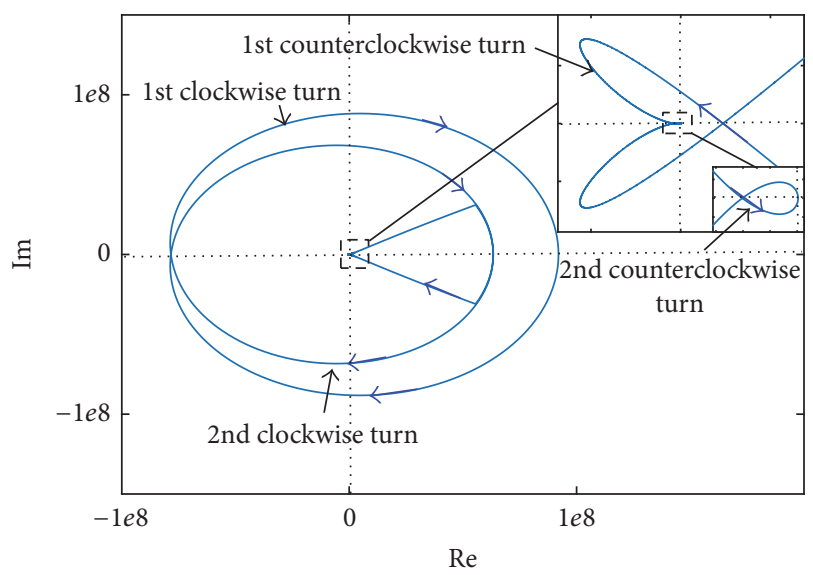

(e) $\tau_{2}=0.008$

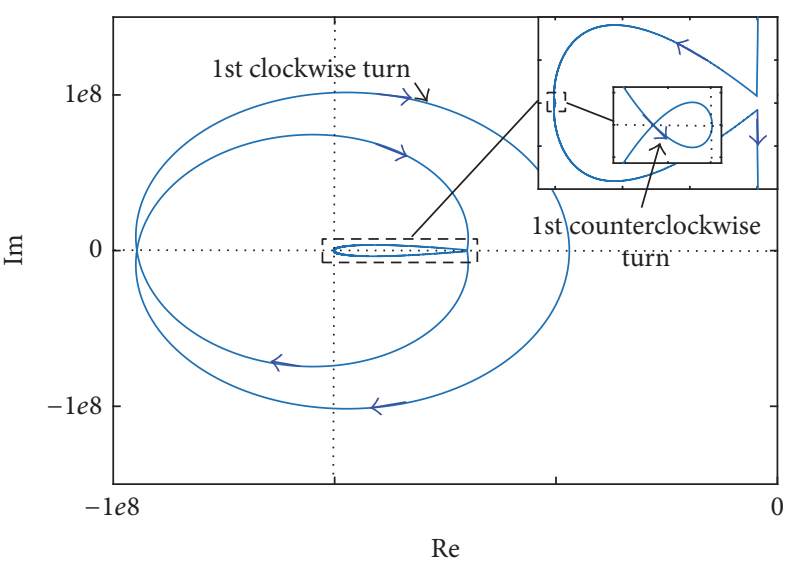

(b) $\tau_{2}=0.001$

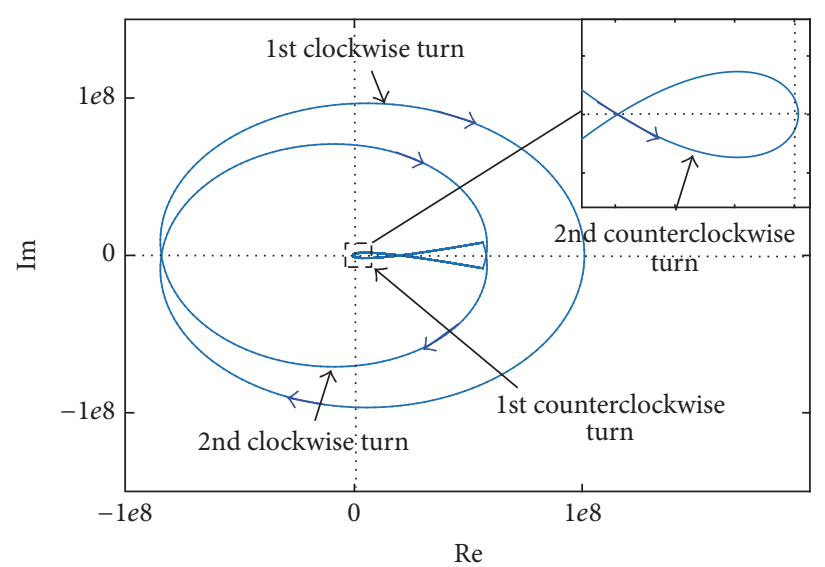

(d) $\tau_{2}=0.003$

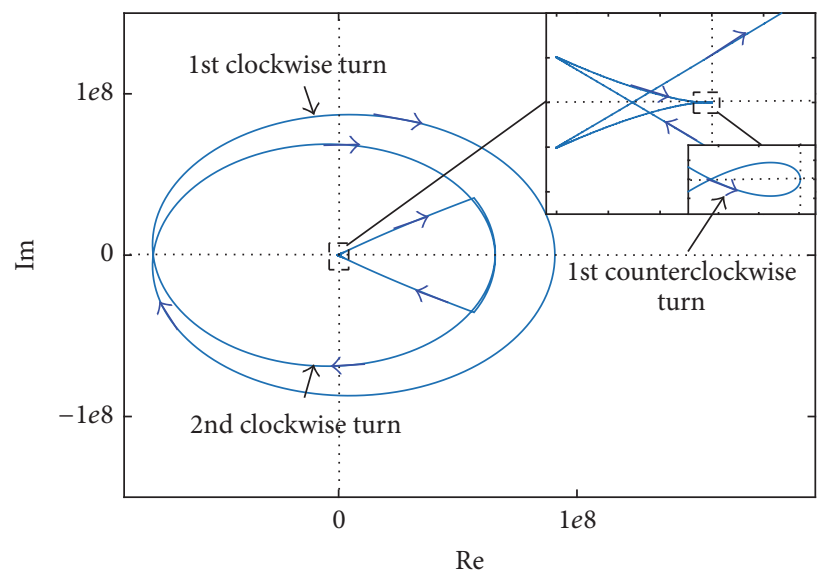

(f) $\tau_{2}=0.009$

FIGURE 6: Mikhailov criterion for quasi polynomial (9) when $\alpha=\beta=0.5$ and $\tau_{2} \in[0,0.009]$.

as is shown in Table 2. From Table 2, it is clear that, due to the wireless communication, the estimation of $\dot{\theta}$ is deteriorated, resulting in a bigger error and decreased closedloop performance when we use this estimation with a state feedback controller.

Remark 3. With the proposed PR controller, the use of the estimation of $\dot{\theta}$ is not required. This is desirable in applications where the estimation of velocity deteriorates the overall performance.

An additional experiment was performed in order to test the robustness against external perturbations. For this aim, several impacts were applied to the pendulum at the time instants $t_{i}, i=1, \ldots, 6$, as can be seen in Figures 10 and 11. 


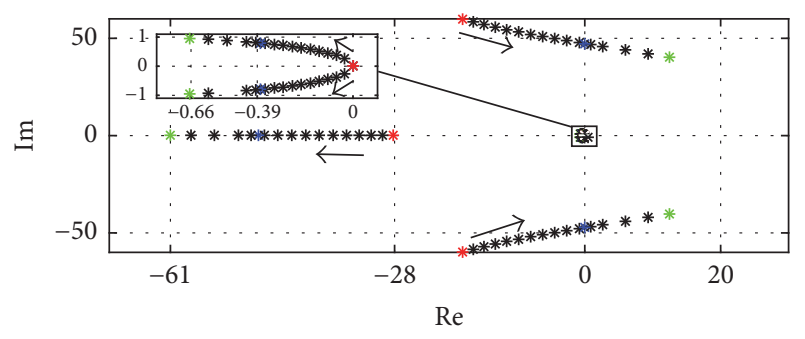

$* \alpha=\beta=0$

* $\alpha=\beta=0.524$

$* \alpha=\beta=0.9$

Figure 7: Root locus of (9) when $\tau_{2}=0.008$ and $\alpha=\beta \in[0,0.9]$.

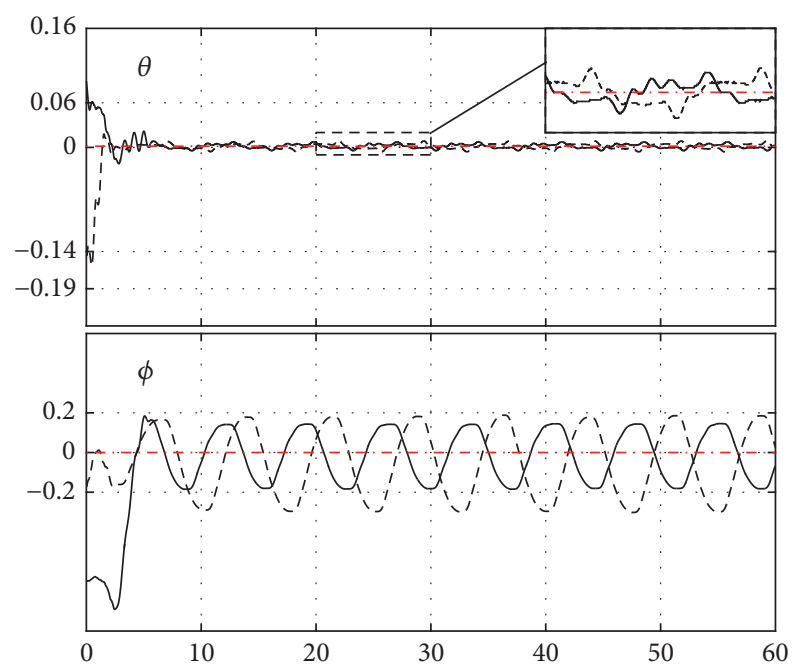

Figure 8: Angular positions ( $\mathrm{rad}) \theta(t)$ and $\phi(t)$ versus time (s): (solid line) PR controller, (dashed line) state feedback controller, and (dashed dotted line) reference.

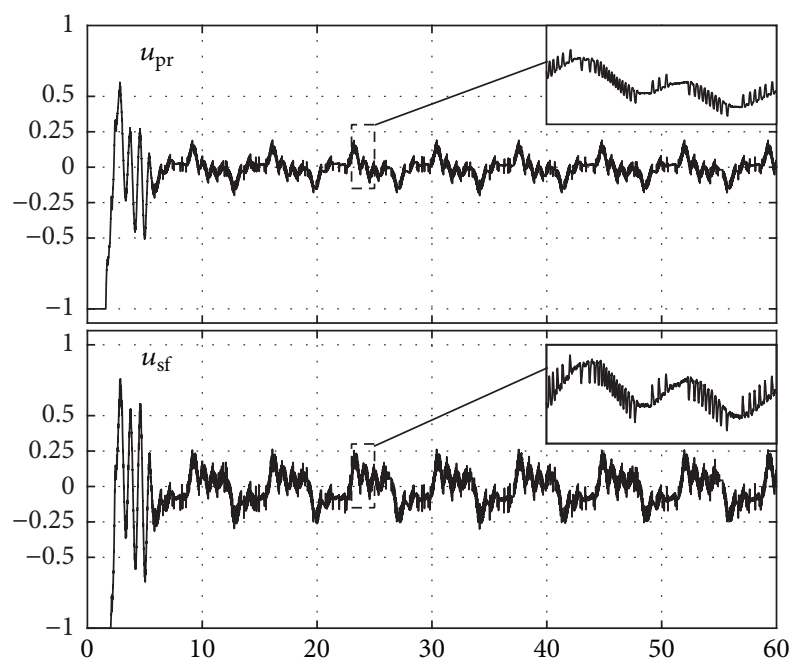

Figure 9: Control actions $(N)$, PR controller $\left(u_{\mathrm{pr}}\right)$, and state feedback controller $\left(u_{\mathrm{sf}}\right)$.
TABLE 2: Table of error comparison.

\begin{tabular}{llccc}
\hline & & \multicolumn{3}{c}{ Differentiators } \\
& & HG1 & HG2 & ST \\
\hline \multirow{2}{*}{ Error $\dot{\theta}$} & Norm 2 & 1.4167 & 1.1556 & 1.349 \\
& Norm inf & 24.9879 & 25.0273 & 10.0679 \\
\multirow{2}{*}{ Error $\dot{\phi}$} & Norm 2 & .3888 & .1292 & .0634 \\
& Norm inf & .8410 & 3.2624 & .5597 \\
\hline
\end{tabular}

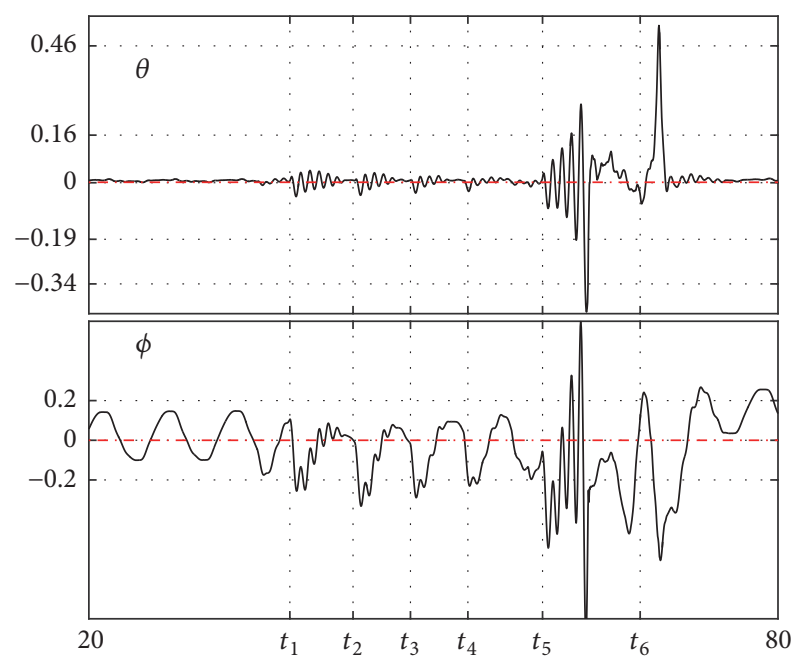

Figure 10: Angular positions ( $\mathrm{rad}) \theta(t)$ and $\phi(t)$ versus time (s) under external perturbations with PR controller.

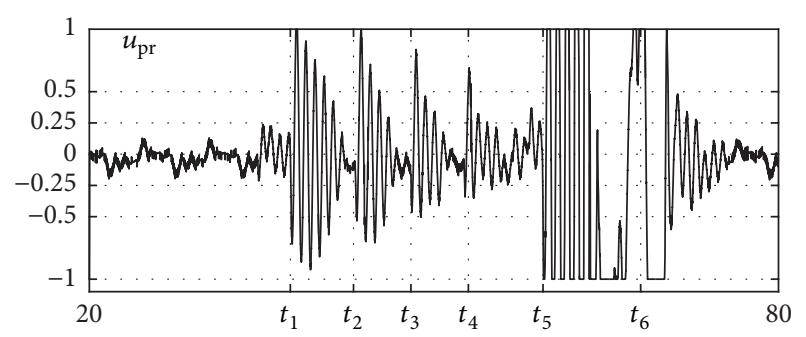

FIGURE 11: Control action $(N)$ under external perturbations with PR controller.

From the experimental results, the recovery of performance with the proposed PR controller is verified. Besides, it is important to highlight that a better transient, a smoother response, and chattering attenuation in the control signal are observed with the proposed approach under the presence of delays in measurements. A video of the recorded experiments is available in the following link: https://www.youtube.com/watch?v=F9QbPVkagwE.

\section{Conclusion}

Presence of time delays, quantization errors of encoders, and errors of the wireless communication make the design of a robust control law difficult. The design and stability analysis of a PR action-based controller for stabilization of the Furuta pendulum under the presence of time delays 
in measurements is introduced in this work. With the proposed approach, the design of observers for the passive variable is avoided simplifying the control algorithm and recovering an acceptable performance without relying on accurate estimates of the derivative for feedback. Since a retarded action is included in the control law, the closedloop dynamics are represented by a characteristic quasi polynomial and the stability analysis was successfully done with Mikhailov's criterion and a root locus of the corresponding quasi polynomial. Furthermore, the stability analysis includes an inherent time delay due to the wireless communication. Besides, a two-step constructive procedure for tuning the four parameters of a controller is presented. Experimental results and a comparison with a state feedback controller show the advantages of the proposed methodology. We are aware that a possible extension of the proposed controller is the addition of a PR controller in the actuated variable as well; however, this is considered as a future work, since the order of the quasi polynomial will increase significantly, losing the scope of the actual contribution. Additionally, extension for a wider class of underactuated mechanical systems and the inclusion of nonlinearities is considered for future work.

\section{Appendix}

\section{A. PR Controller for a Second-Order Plant}

Consider the next second-order system,

$$
\ddot{\theta}(t)-b \dot{\theta}-a \theta(t)=d U(t),
$$

and the PR control law,

$$
U(t)=k_{p} \theta(t)-k_{r} \theta(t-\tau),
$$

where the design parameters are as follows: $k_{p}$ is the proportional gain, $k_{r}$ is the retarded gain, and $\tau$ is the time delay. Thus, the characteristic quasi polynomial of closedloop system (A.1)-(A.2) is

$$
p\left(s, k_{p}, k_{r}, \tau\right)=s^{2}-b s-\left(a+d k_{p}\right)+d k_{r} e^{s \tau} .
$$

We say that the triplet $\left(k_{p}, k_{r}, \tau\right) \sigma$-stabilizes quasi polynomial (A.3) if

$$
\alpha_{0} \leq \sigma, \quad \sigma \in \mathbb{R}^{+},
$$

with $\alpha_{0}=\sup \left\{\operatorname{Re}\left\{s_{j}\right\}: p\left(s_{j}, k_{p}, k_{r}, \tau\right)=0, s_{j} \in \mathbb{C}\right\}$, where $\operatorname{Re}\left\{s_{j}\right\}$ is the real part of $s_{j}, j=1,2, \ldots, \infty$. In [16], the parameter space $\left(k_{p}, k_{r}, \tau\right)$ of control law [13] to $\sigma$-stabilize system [12] is determined.

Lemma A.1 (see [16]). Let $k_{p}$ be such that $-d k_{p}>a+(b / 2)^{2}$ and $\sigma \in\left[-b / 2, \sigma^{*}\right]$, where

$$
\sigma^{*}=-\frac{b}{2}+\sqrt{-a-(b / 2)^{2}-d k_{p}}
$$

Then, the parametric equations delimiting the regions of $\sigma$ stabilizability of closed-loop system (A.1)-(A.2) are given by the following:

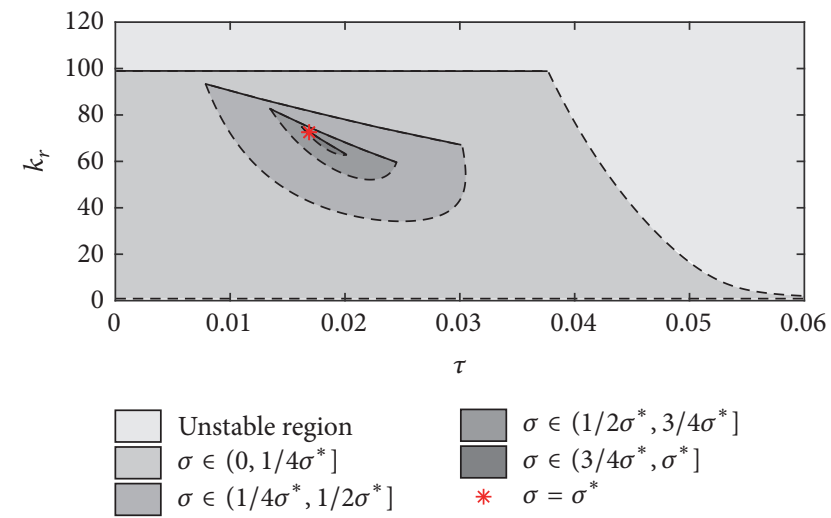

FIGURE 12: $\sigma$-Stability regions of closed-loop system (A.1)-(A.2). Here, $\sigma^{*}=59.4573, \tau^{*}=0.0169$, and $k_{r}^{*}=72.4822$.

Lower Boundary

$$
k_{r}(\omega, \tau)=-\frac{\left(\sigma^{2}+\sigma b-a-d k_{p}\right)}{\left(d e^{\tau \sigma}\right)},
$$

for $\tau$ defined as for the upper boundary.

Upper Boundary

$$
\begin{aligned}
& \tau(\omega) \\
& =\left\{\begin{array}{l}
\frac{1}{\omega} \cot ^{-1}\left(\frac{\left(\sigma^{2}+\sigma b-\omega^{2}-a-d k_{p}\right)}{(2(\sigma+(b / 2)) \omega)}\right), \quad \omega \in\left(0, \omega_{e}\right], \\
\frac{1}{\omega} \cot ^{-1}\left(\frac{\left(\sigma^{2}+\sigma b-\omega^{2}-a-d k_{p}\right)}{(2(\sigma+(b / 2)) \omega)}\right)+\frac{\pi}{\omega}, \quad \omega \in\left(\omega_{e}, \omega_{0}\right],
\end{array}\right. \\
& k_{r}(\omega, \tau)=-\left(\frac{2 \omega(\sigma+(b / 2))}{\left(d e^{\sigma \tau} \sin (\omega \tau)\right)}\right), \\
& \omega_{0}=\sqrt{-2\left[a+\sigma^{2}+\sigma b+d k_{p}-b^{2}\right]}, \\
& \omega_{e}=\min \left\{\omega_{0}, \omega_{\pi}\right\}, \\
& \omega_{\pi}=\sqrt{\sigma^{2}+\sigma b-a-d k_{p} .}
\end{aligned}
$$

In Figure 12, the upper boundary (solid line) and lower bounds (dashed line) using $k_{p}=100$ and the parameters given in Table 1 are shown. These bounds delimit the $\sigma$ stability regions for quasi polynomials of the form (A.3).

Also, note that these regions collapse into a single point (red asterisk) which correspond to the unique maximum achievable decay $\sigma^{*}$. The following result characterized analytically this point.

Lemma A.2 (see [16]). Let $k_{p}$ be such that $-k_{p}>+(b / 2)^{2}$. Then, the maximum exponential decay $\sigma^{*}$ of the closed-loop system (A.1)-(A.2) is given in (A.5). Moreover, the values 

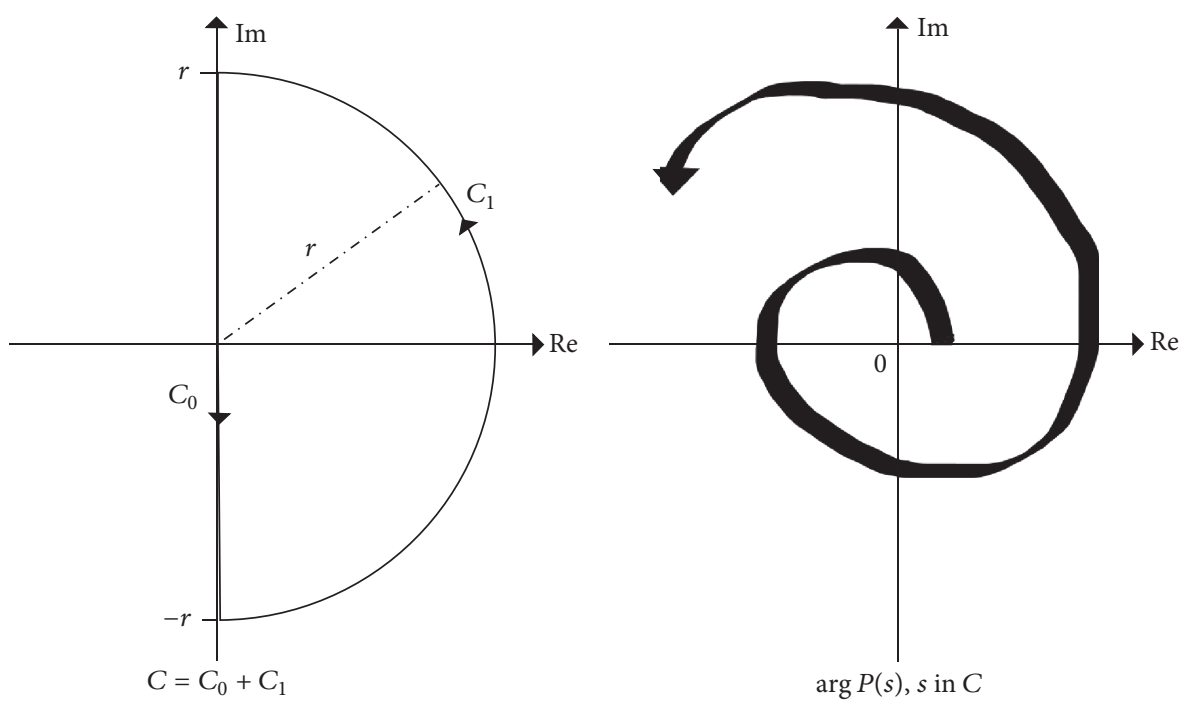

Figure 13: Example of $\Delta_{C} \arg \{P(s)\}$ along a curve $C_{0}$.

$\left(k_{r}^{*}, \tau^{*}\right)$ that $\sigma$-stabilize system (A.1)-(A.2) with the best possible exponential decay $\sigma^{*}$ are

$$
\begin{aligned}
\tau^{*} & =\left(\frac{\left(2 \sigma^{*}+b\right)}{\left(\left(\sigma^{*}\right)^{2}+b \sigma^{*}-a-d k_{p}\right)}\right), \\
k_{r}^{*} & =-\left(\frac{\left(2 \sigma^{*}+b\right)}{\left(d \tau^{*} e^{\sigma^{*} \tau^{*}}\right)}\right) .
\end{aligned}
$$

For illustrative purposes, for Figure 12, we take $k_{p}=100$.

\section{B. Summary of Mikhailov's Criterion}

Stability conditions stated in terms of hodographs (frequency plots) have a long history. In their most general form, these conditions were given by Cauchy's principle of the argument around 1829. The graphical use of the argument principle was introduced to the engineering community by Nyquist in 1932 and Mikhailov in 1938 for linear time-independent systems; see $[36,37,52-54]$ for extensions to the case with time delays. Mikhailov's criterion gives necessary and sufficient conditions for the stability of a quasi polynomial for a timedelay system. Let us formulate one of some possible versions of this criterion.

Theorem B.1. Consider the following quasi polynomial for a time-delay system:

$$
P(s)=\sum_{k=0}^{m} A_{k}(s) e^{-h_{i} s}, \quad s \in C \subset \mathbb{C},
$$

where $C$ is a closed curve in the complex plane $\mathbb{C}, 0=h_{0}<h_{1}<$ $\cdots<h_{m}, A_{K}(s)=\sum_{j=0}^{n_{k}} a_{j k} s^{j}, a_{j k} \in \mathbb{R}, n_{0} \geq 1$, and $n_{k} \leq n_{0}$, for $k=1, \ldots, m$. If $P(s)$ has no roots on the curve $\mathbb{C}$, then the number $N$ of its roots inside of $C$ is

$$
N=\frac{1}{2 \pi} \Delta_{C} \arg \{P(s)\},
$$

where $\Delta_{C} \arg \{P(s)\}$ denotes the changes of the argument of $P$ along $C$.

Immediately, the above theorem is used to determine stability or instability of quasi polynomials of Section 3, exploiting the well-known fact that the number of unstable roots is always finite (see [55], page 9) and therefore can be trapped by a large enough semicircle in the right-half plane.

Let $C=C_{0}+C_{1}$ denote one closed curve in the complex plane, as depicted in Figure 13. Here, $C_{0}$ is the interval $[-r, r]$ on the imaginary axis and $C_{1}$ is the semicircle of the radius $r$ in the right-half (left-half) complex plane. It follows from the above theorem that

$$
N=1 /(2 \pi)\left(\Delta_{C_{0}} \arg \{P(s)\}+\Delta_{C_{1}} \arg \{P(s)\}\right),
$$

where $\Delta_{C_{0}} \arg \{P(s)\}$ and $\Delta_{C_{1}} \arg \{P(s)\}$ denote the changes of argument of $P$ along $C_{0}$ and $C_{1}$, respectively. Assuming that the radius $r$ is sufficiently large, to claim stability, one must just verify that $N=0$, that is, verify that the number of the turns around the origin in the clockwise direction is equal to the number of turns in the counterclockwise direction.

It should be noted, however, that application of Mikhailov's method in the case of quasi polynomials must be done with great care, since there is typically no monotonicity of rotation of the curve along $C_{0}$, as in Figure 13, which can be proven only for the case of polynomials with a finite number of roots.

\section{Nomenclature}

$\theta$ : Angular position of the pendulum

$\phi$ : Angle of the rotational rod

$m_{1}$ : Mass of the rotational rod

$m_{2}$ : Pendulum mass

$J_{1}$ : Moment of inertia of the rod

$J_{2}$ : Moment of inertia of the pendulum

$L_{1}$ : Length of the horizontal rod 
$L_{2}$ : Pendulum length

$l_{1}$ : Distance to rod center of gravity

$l_{2}$ : Distance to pendulum center of gravity

$g$ : Gravity acceleration

$M_{a}$ : Induced torque.

\section{Conflicts of Interest}

The authors declare that there are no conflicts of interest regarding the publication of this paper.

\section{References}

[1] M. Spong, "Partial feedback linearization of underactuated mechanical systems," in Proceedings of the IEEE/RSJ/GI International Conference on Intelligent Robots and Systems (IROS'94), pp. 314-321, IEEE, Munich, Germany.

[2] M. Reyhanoglu, A. van der Schaft, N. H. McClamroch, and I. Kolmanovsky, "Dynamics and control of a class of underactuated mechanical systems," Institute of Electrical and Electronics Engineers Transactions on Automatic Control, vol. 44, no. 9, pp. 1663-1671, 1999.

[3] A. M. Bloch, N. E. Leonard, and J. E. Marsden, "Controlled Lagrangians and the stabilization of mechanical systems i: the first matching theorem," Institute of Electrical and Electronics Engineers Transactions on Automatic Control, vol. 45, no. 12, pp. 2253-2270, 2000.

[4] R. Ortega, M. W. Spong, F. Gómez-Estern, and G. Blankenstein, "Stabilization of a class of underactuated mechanical systems via interconnection and damping assignment," Institute of Electrical and Electronics Engineers Transactions on Automatic Control, vol. 47, no. 8, pp. 1218-1233, 2002.

[5] R. Olfati-Saber, "Normal forms for underactuated mechanical systems with symmetry," Institute of Electrical and Electronics Engineers Transactions on Automatic Control, vol. 47, no. 2, pp. 305-308, 2002.

[6] J. A. Acosta, R. Ortega, A. Astolfi, and A. D. Mahindrakar, "Interconnection and damping assignment passivity-based control of mechanical systems with underactuation degree one," Institute of Electrical and Electronics Engineers Transactions on Automatic Control, vol. 50, no. 12, pp. 1936-1955, 2005.

[7] A. S. Shiriaev, L. B. Freidovich, and S. V. Gusev, "Transverse linearization for controlled mechanical systems with several passive degrees of freedom," Institute of Electrical and Electronics Engineers Transactions on Automatic Control, vol. 55, no. 4, pp. 893-906, 2010.

[8] Y. Liu and H. Yu, "A survey of underactuated mechanical systems," IET Control Theory \& Applications, vol. 7, no. 7, pp. 921-935, 2013.

[9] A. S. Shiriaev, L. B. Freidovich, and M. W. Spong, "Controlled invariants and trajectory planning for underactuated mechanical systems," Institute of Electrical and Electronics Engineers Transactions on Automatic Control, vol. 59, no. 9, pp. 2555-2561, 2014.

[10] PendCon, "PendCon Advanced: Rotary Pendulum, Germany".

[11] N. J. Ploplys, P. A. Kawka, and A. G. Alleyne, "Closed-loop control over wireless networks," IEEE Control Systems Magazine, vol. 24, no. 3, pp. 58-71, 2004.

[12] V. C. Gungor and G. P. Hancke, "Industrial wireless sensor networks: challenges, design principles, and technical approaches,"
IEEE Transactions on Industrial Electronics, vol. 56, no. 10, pp. 4258-4265, 2009.

[13] D.-I. Curiac, "Towards wireless sensor, actuator and robot networks: conceptual framework, challenges and perspectives," Journal of Network and Computer Applications, vol. 63, no. 1, pp. 14-23, 2009.

[14] A. Levant, "Higher-order sliding modes, differentiation and output-feedback control," International Journal of Control, vol. 76, no. 9-10, pp. 924-941, 2003.

[15] H. K. Khalil and L. Praly, "High-gain observers in nonlinear feedback control," International Journal of Robust and Nonlinear Control, vol. 24, no. 6, pp. 993-1015, 2014.

[16] R. Villafuerte, S. Mondié, and R. Garrido, "Tuning of proportional retarded controllers: theory and experiments," IEEE Transactions on Control Systems Technology, vol. 21, no. 3, pp. 983-990, 2013.

[17] T. Ortega, R. Villafuerte, C. Vázquez, and L. Freidovich, "Performance without tweaking differentiators via a PR controller: furuta pendulum case study," in Proceedings of the 2016 IEEE International Conference on Robotics and Automation, (ICRA '16), pp. 3777-3782, IEEE, Stockholm, Sweden, May 2016.

[18] I. H. Suh and Z. Bien, "Proportional minus delay controller," Institute of Electrical and Electronics Engineers Transactions on Automatic Control, vol. 24, no. 2, pp. 370-372, 1979.

[19] I. H. Suh and Z. Bien, "Use of time-delay actions in the controller design," IEEE Transactions on Automatic Control, vol. 25 , no. 3, pp. 600-603, 1980.

[20] G. M. Swisher and T. Shing, "Design of proportional-minusdelay action feedback controllers for second- and third-order systems," in Proceedings of the 1988 American Control Conference, pp. 254-260, 1998.

[21] C. Abadallah, P. Dorato, J. Benites-Read, and R. Byrne, "Delayed positive feedback can stailize oscillatory systems," in Proceedings of the American Control Conference, 1993.

[22] Q. Zhong and H. Li, "A delay-type pid controller," in IFAC Proceedings Volumes, vol. 35 of 15th Triennial World Congress, pp. 265-270, Barcelona, Spain, 1 edition, 2002.

[23] S.-I. Niculescu and W. Michiels, "Stabilizing a chain of integrators using multiple delays," Institute of Electrical and Electronics Engineers Transactions on Automatic Control, vol. 49, no. 5, pp. 802-807, 2004.

[24] V. L. Kharitonov, S.-I. Niculescu, J. Moreno, and W. Michiels, "Static output feedback stabilization: necessary conditions for multiple delay controllers," Institute of Electrical and Electronics Engineers Transactions on Automatic Control, vol. 50, no. 1, pp. 82-86, 2005.

[25] M. Krstic, Delay Compensation for Nonlinear, Adaptative, and PDE Systems, Birkhäuser, Basel, Switzerland, 2009.

[26] N. Olgac and M. E. Cavdaroglu, "Full-state feedback controller design with "delay scheduling" for cart-and-pendulum dynamics," Mechatronics, vol. 21, no. 1, pp. 38-47, 2011.

[27] F. Mazenc and M. Malisoff, "New control design for bounded backstepping under input delays," Automatica, vol. 66, pp. 4855, 2016.

[28] E. Fridman, Introduction to Time-delay Systems: Analysis and Control, Springer, Berlin, Germany, 2014.

[29] K. Gu, V. L. Kharitonov, and J. Chen, Stability of Time Delay Systems, Control eniginnering, Sprnger, Berlin, Germany, 2003.

[30] K. Engelborghs, T. Luzyanina, and D. Roose, "Numerical bifurcation analysis of delay differential equations using DDEBIFTOOL," IEEE Transactions on Automatic Control, vol. 54, no. 1, pp. 171-177, 2002. 
[31] M. Dellnitz, O. Schutze, and Q. Zheng, "Locating all the zeros of an analytic function in one complex variable," Journal of Computational and Applied Mathematics, vol. 138, no. 2, pp. 325-333, 2002.

[32] T. Vyhlídal and P. Zítek, "Mapping based algorithm for largescale computation of quasi-polynomial zeros," IEEE Transactions on Automatic Control, vol. 28, no. 1, pp. 1-21, 2009.

[33] K. Aström and K. Furuta, "Swining up a pendulum by energy control," FAC 13th World Congress, 1996, San Francisco, USA.

[34] M. Ramírez-Neria, H. Sira-Ramírez, R. Garrido-Moctezuma, and A. Luviano-Juárez, "Linear active disturbance rejection control of underactuated systems: the case of the Furuta pendulum," ISA Transactions, vol. 53, no. 4, pp. 920-928, 2014.

[35] A. Mikhailov, "Method of harmonic analysis in control theory," Avtomatika i Telemekhanika, vol. 3, no. 1, pp. 27-81, 1938.

[36] I. Kabaknov, "Concerning the control process for the steam pressure," Inzenernyj Sobrnik, vol. 2, no. 2, pp. 27-60, 1946 (Russian).

[37] U. Forys, "Biological delay systems and the mikhailov criterion of stability," Journal of Biological Systems, vol. 12, no. 1, pp. 45$60,2004$.

[38] J. Neimark, "D-subdivisions and spaces of quasi-polynomials," Prikl. Mat. Meh, vol. 13, no. 1, pp. 349-380, 1949.

[39] W. Michiels and S.-I. Niculescu, "Stability, control, and computation for time-delay systems: an eigenvalue-based approach," SIAM: Advances in Design and Control, vol. 27, 2014.

[40] X.-G. Li, S.-I. Niculescu, and A. Cela, "Analytic curve frequency-sweeping stability tests for systems with commensurate delays," Springer Briefs in Electrical and Computer Engineering: Control, Automation and Robotics, 2015.

[41] K. Cooke and P. Van Den Driessche, "On the zeros of some elementary transcendental functions," American Mathematical Society Translations, vol. 2, no. 1, pp. 95-110, 1955.

[42] K. L. Cooke and P. Van den Driessche, "On zeroes of some transcendental equations," Funkcialaj Ekvacioj, vol. 29, no. 1, pp. 77-90, 1986.

[43] V. L. Kharitonov and A. P. Zhabko, "Robust stability of timedelay systems," Institute of Electrical and Electronics Engineers Transactions on Automatic Control, vol. 39, no. 12, pp. 23882397, 1994.

[44] N. Olgac and R. Sipahi, "An exact method for the stability analysis of time-delayed linear time-invariant (LTI) systems," Institute of Electrical and Electronics Engineers Transactions on Automatic Control, vol. 47, no. 5, pp. 793-797, 2002.

[45] L. Freidovich, A. Shiriaev, F. Gordillo, F. Gómez-Estern, and J. Aracil, "Partial-energy-shaping control for orbital stabilization of high-frequency oscillations of the furuta pendulum," IEEE Transactions on Control Systems Technology, vol. 17, no. 4, pp. 853-858, 2009.

[46] P. X. La Hera, L. B. Freidovich, A. S. Shiriaev, and U. Mettin, "New approach for swinging up the furuta pendulum: theory and experiments," Mechatronics, vol. 19, no. 8, pp. 1240-1250, 2009.

[47] S. Mori, H. Nishihara, and K. Furuta, "Control of unstable systems," International Journal of Control, vol. 23, no. 5, pp. 673692, 1976.

[48] A. Ferreira, F. J. Bejarano, and L. M. Fridman, "Robust control with exact uncertainties compensation: with or without chattering?" IEEE Transactions on Control Systems Technology, vol. 19, no. 5, pp. 969-975, 2011.
[49] J. Aracil, J. Á. Acosta, and F. Gordillon, "A nonlinear hybrid controller for swinging-up and stabilizing the Furuta pendulum," Control Engineering Practice, vol. 21, no. 8, pp. 989-993, 2013.

[50] A. Levant, "Robust exact differentiation via sliding mode technique," Automatica, vol. 34, no. 3, pp. 379-384, 1998.

[51] L. Biagiotti and C. Melchiorri, Trajectory Planning for Automatic Machines and Robots, Springer, Berlin, Germany, 2008.

[52] Y. Tzypkin, "Stability of systems with delayed feedback," Automation and Remote Control, vol. 7, no. 2-3, pp. 107-129, 1946.

[53] A. Drozd and Z. Filer, "Application of the Mikhailov criterion to the study of stability of systems with delay," Journal of Computer and Systems Sociences International, vol. 38, no. 3, pp. 339-342, 1998.

[54] L. Pekar, R. Prokop, and R. Maturu, "A stability test for control systems with delays based on the nyquist criterion," International Journal of Mathematical Models and Methods in Applied Sciences, vol. 5, no. 7, pp. 1213-1224, 2011.

[55] W. Michiels and S.-I. Niculescu, "Stability and stailization of time-delay systems advances in design and control," Society for Industrial and Applied Mathematics, 2007. 


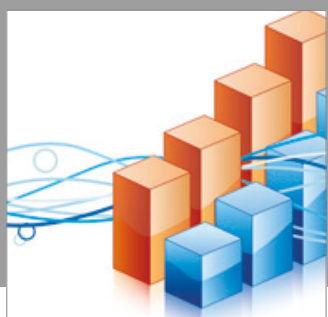

Advances in

Operations Research

vatersals

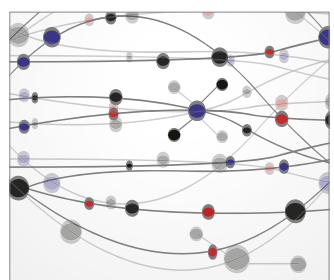

\section{The Scientific} World Journal
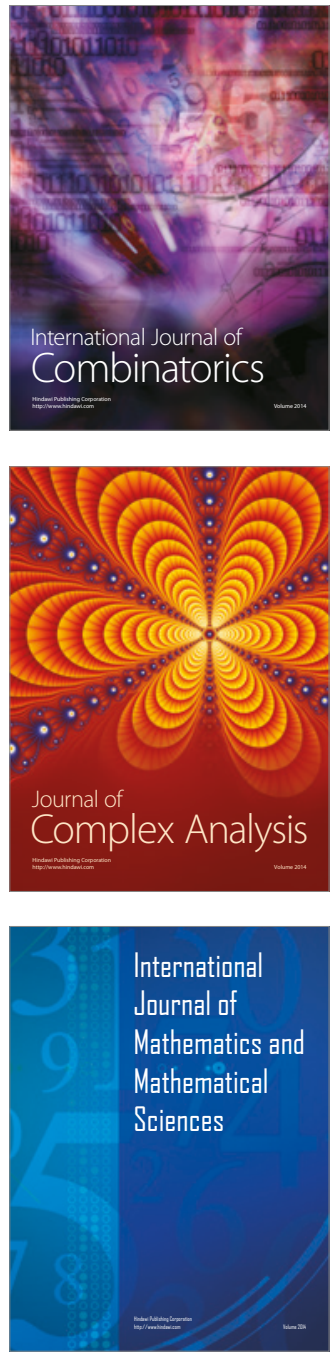
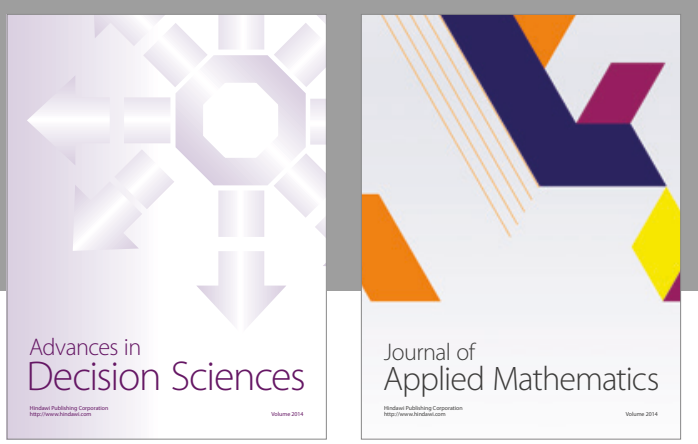

Algebra

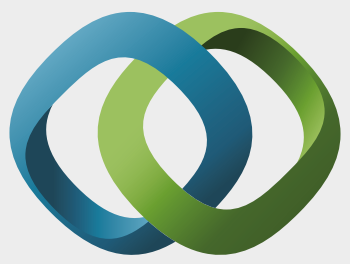

\section{Hindawi}

Submit your manuscripts at

https://www.hindawi.com
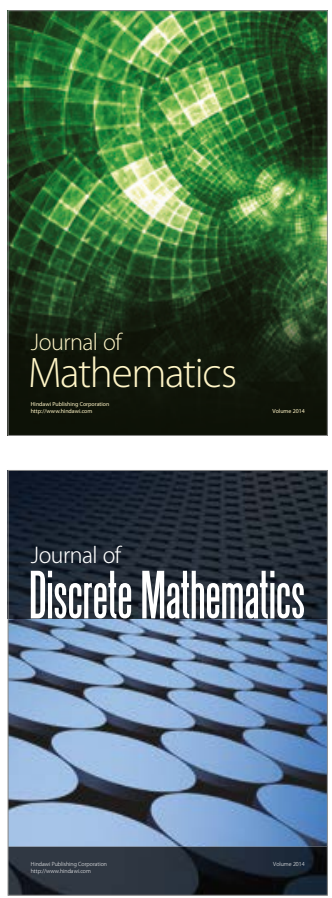

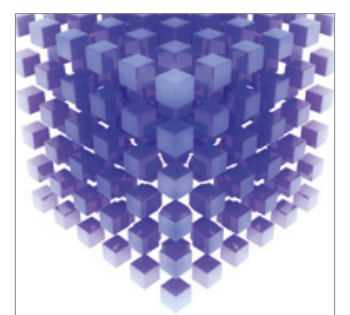

Mathematical Problems in Engineering
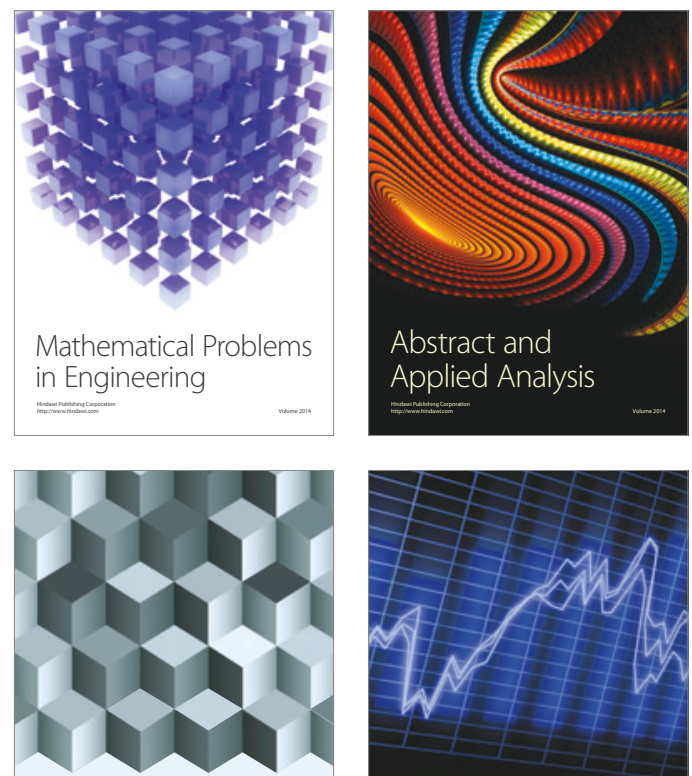

Journal of

Function Spaces

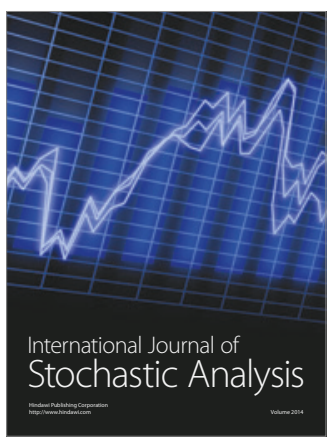

Probability and Statistics
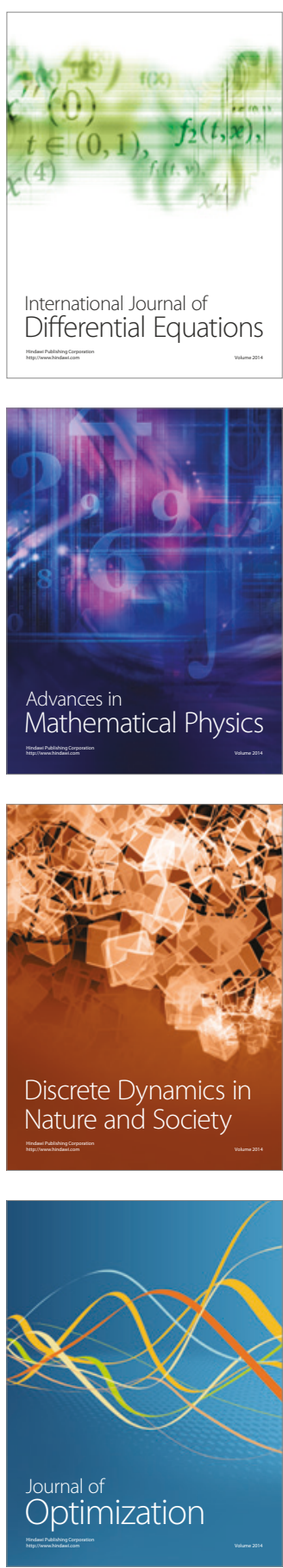